\title{
Understanding the Communicative and Social Processes of Engineering Ethics in Diverse Design Teams
}

\section{Dr. Carla B. Zoltowski, Purdue University, West Lafayette}

Carla B. Zoltowski, Ph.D., is Co-Director of the EPICS Program at Purdue University. She received her B.S. and M.S. in electrical engineering and Ph.D. in engineering education, all from Purdue University. She has served as a lecturer in Purdue's School of Electrical and Computer Engineering. Dr. Zoltowski's academic and research interests include human-centered design learning and assessment, service-learning, ethical reasoning development and assessment, leadership, and assistive technology.

\section{Prof. Patrice Marie Buzzanell, Purdue University, West Lafayette}

Patrice M. Buzzanell is a Professor in the Brian Lamb School of Communication and the School of Engineering Education (courtesy) at Purdue University. Editor of three books and author of over 150 articles and chapters, her research centers on the intersections of career, gender communication, leadership, and resilience. Fellow and past president of the International Communication Association, she has received numerous awards for her research, teaching/mentoring, and engagement. She is working on Purdue-ADVANCE initiatives for institutional change, the Transforming Lives Building Global Communities (TLBGC) team in Ghana through EPICS, and individual engineering ethical development and team ethical climate scales as well as everyday negotiations of ethics in design through NSF funding as Co-PI. [Email: buzzanel@purdue.edu]

\section{Dr. William C. Oakes, Purdue University, West Lafayette}

William (Bill) Oakes is the Director of the EPICS Program and one of the founding faculty members of the School of Engineering Education at Purdue University. He has held courtesy appointments in Mechanical, Environmental and Ecological Engineering as well as Curriculum and Instruction in the College of Education. He is a registered professional engineer and on the NSPE board for Professional Engineers in Higher Education. He has been active in ASEE serving in the FPD, CIP and ERM. He is the past chair of the IN/IL section. He is a fellow of the Teaching Academy and listed in the Book of Great Teachers at Purdue University./ He was the first engineering faculty member to receive the national Campus Compact Thomas Ehrlich Faculty Award for Service-Learning. He was a co-recipient of the National Academy of Engineering's Bernard Gordon Prize for Innovation in Engineering and Technology Education and the recipient of the National Society of Professional Engineers' Educational Excellence Award and the ASEE Chester Carlson Award. He is a fellow of the American Society for Engineering Education and the National Society of Professional Engineers.

\section{Megan Kenny Feister, Purdue University}

Megan is a fourth year doctoral candidate in the Brian Lamb School of Communication at Purdue University pursuing a $\mathrm{PhD}$ in Organizational Communication with a minor in mixed methods. Her research focuses on engineering education, design, organizational identity, identification and socialization, team communication, innovation, and technology. She is currently working on an NSF grant examining ethical reasoning and decision-making in engineering project teams, and examining the relationship between teams and individuals in engineering design from a social constructionist and social network perspective.

\section{David Torres, Purdue University}

David is a first year doctoral student in the Brian Lamb School of Communication at Purdue University pursuing a $\mathrm{PhD}$ in Organizational Communication with a minor in data analysis and research methodology. His research interests reside at the intersection of organizational communication, organizational ethics, social network analysis, identity and identification, and leadership development. 


\section{Understanding the Communicative and Social Processes of Engineering Ethics in Diverse Design Teams}

\section{Introduction}

As engineering, and specifically engineering design, is increasingly understood to be a social activity, engineering education's understanding of ethics needs to reflect this developing awareness. Within engineering and design teams, engineering educators are concerned not only with how individual students develop ethically, but also how everyday ethical decision-making emerges during team interactions and becomes integrated in design solutions. Furthermore, these ethical decisions often do not present themselves as traditional dilemmas, but are issues that are confronted in the everyday process of design, and are influenced by team members' cultural and disciplinary backgrounds and the ethical climates of the team and the organization.

In considering engineering ethics education in this context, we can draw from the extensive scholarship on group communication. This body of literature suggests that team member interactions and communication have a major impact on a team's decision-making abilities, as well as the information that is discussed during the problem-solving process ${ }^{1-4}$. Therefore, this project seeks to understand how everyday ethical decision-making is embedded in the processes and interactions of diverse engineering design team and their recognition of the long-term design consequences of their solutions and it guided by the following four research questions:

RQ1: How is "everyday ethics" experience and communicatively constituted by students in multidisciplinary engineering design teams?

RQ2: How do individual team members influence the team's organization and behaviors regarding ethics in team decision-making? How do the disciplinary diversity and cultural influences shape team member interactions in ethical decision-making?

RQ3: How do team organization and team member interactions shape team ethical decision-making behaviors and team ethical climate?

RQ4: What characteristics of team member interactions and team organizational structure can encourage teams in the development of ethical decision-making processes in multidisciplinary engineering design teams?

To answer our research questions, this study combines social network analysis (SNA) with structuration theory to examine the structure of project teams while also examining the institutional and contextual factors that contribute to team climate, and to the development of group norms that affect team interactions. SNA is a type of analysis that enables researchers to examine the relationships among members of a given system or group. In contrast to the "organizational chart" that might show how communication is supposed to flow within the organization, network analysis shows the actual communication and relationships that emerge within the organization or team. Structuration accounts for the influence of institutional factors such as rules or norms of what is "acceptable" or "appropriate" behavior within a specific social context, while also affording the actors within that context agency to influence those institutional factors. Primary data sources include a series of interviews and videotaped participatory 
observations, as well as the social network analysis survey. In the paper, we describe the study frameworks and the specific methods employed in the project to date.

\section{Motivation}

This project builds upon prior NSF-funded projects that examined individual ethical decisionmaking and ethical team climate in multidisciplinary project teams in an engineering context. Our efforts centered on the development of two instruments, one to assess individual engineering ethical reasoning, and the other to assess team ethical climate. As part of the prior project, we conducted 51 interviews with students on these teams to probe the concepts examined in these instruments with the intent of qualitatively validating these instruments and providing greater depth and context ${ }^{5}$. In our analysis of these interviews, we uncovered consistent themes indicating that students did not identify issues such as a breach of a non-disclosure or confidentiality agreements or of overtly unsafe design elements as having ethical implications or decision making unless explicitly directed to frame such issues as ethical. In addition, students were not aware that they were engaged in smaller decisions that have ethical implications such as tensions among efficiencies or compliance with engineering standards, nor that they participated in ongoing ethical decision making during their interactions with each other and with their project partners or clients. Furthermore, they often did not consider how the context in which their design was being developed and for whom they were designing included ethical considerations.

Although students may realize neither that they are engaging in ethical processes nor how the context in which they work shapes and is shaped by their decisions, we take as a starting point that teams and individuals are engaged in ethical deliberations on an ongoing basis throughout the everyday engineering design process. Our project seeks to understand how such interactive processes occur in terms of the specific network structures and contexts of engineering projects within particular institutional programs; our project can inform best practices for engineering education to translate ongoing ethical decision-making processes into practice ${ }^{6-9}$. The following sections describe the frameworks from ethics, design, and communication that inform our study.

\section{Kohlberg's Moral Development Theory}

Kohlberg's moral development theory (and Neo-Kohlbergian revisions) ${ }^{10-11}$ have been widely used to understand and assess moral reasoning in a variety of professional fields (e.g., science, engineering, medicine, and business) across cultures. From the perspective of moral cognition, Kohlberg's theory attempts to understand how people reason morally and on what values their reasoning processes are based.

Neo-Kohlbergian scholars divide moral development into three schemas. The first schema, preconventional, is concerned with a predominantly self-interested orientation. The second schema, conventional, is based on concerns for external factors (other people and authoritative rules/orders). The third schema, postconventional, builds ethical reasoning on universal norms and values (e.g., justice, human rights) that are concerned with and good for everyone in the world. Moral values and principles are not unquestionably accepted but subject to critique and reflection. Those who reason at this level have the highest level of moral development compared to people at the two earlier levels. Although individual ethical reasoning is necessary but not 
sufficient for moral behavior in team contexts, a neo-Kohlbergian approach does help build the foundation for understanding the ethics in team communication and interactions and is a fundamental way of understanding the (ethical and cultural) diversity of team climate and structure.

Furthermore, postconventional thinking views ethical reasoning as a process based on social arrangements. Applied to the team contexts, "if social arrangements meet certain procedural norms the resulting decisions are considered acceptable"12 (p. 104). These norms enable members to maintain ethical interactions in team structures and factor into everyday ethics.

\section{Everyday Ethics}

Scholarship in science and technology studies (STS) has shown that engineering design is a context in which ethical issues arise on a day-to-day basis or in what is called "everyday context"13. In engineering design, a "usual" impression is that not much engineering designing contains what is normally called "ethical dilemmas" in ethics classes and textbooks. For engineering designers, it is often the case that when looking back, "after things turned out nasty", reasoning originally unrelated to ethics then turns out to be ethical reasoning after all ${ }^{14}$ (p. 514). On the whole engineering design might not seem to be specifically about what we would traditionally consider to be "ethical issues," even though the products of an engineering design process, and especially the use of those products, undoubtedly is ${ }^{14}$ and any agreement achieved or decision made in the design process may result in potential social and ethical impacts.

In this sense, traditional engineering ethics has been criticized by STS scholars as "an externalist approach" to technology where ethics is an external force acting upon design or a "check list" that focuses on "the outcomes of processes of technology development rather than on the internal dynamic of these processes"13 (p. 224, emphasis added). STS scholars advocate an internalist approach that attempts to open the "black box" of technology to complement a traditional externalist approach to engineering ethics. Ethical reflection during the design process requires anticipation of the future role of technologies-in-design in their use context ${ }^{15}$, where ethics is an indispensable component that is possible to be combined with other technical components in design. Besides avoiding doing harm and preventing negative effects of technology emphasized by traditional "preventative/passive ethics", the everyday ethics approach also focuses on active responsibility of the effects of technology.

\section{Design Context}

Design has been characterized by many different "design process" models ${ }^{16-20}$ and definitions ${ }^{21-}$

23. The many design definitions and processes reflect different design approaches, philosophies, and values. For example, technology-centered design has been defined as a process in which the designers or their clients make design decisions which are imposed on the intended users ${ }^{24-25}$, whereas human-centered design have human beings as central in the process, involve users throughout the design process, and seek to understand them holistically ${ }^{26}$. In the context of design, there are many different values, such as innovation or a primary concern for safety, that guide design decisions and processes, and can impact how designers think about the ethical issues related to their designs and the implications of their "everyday" ethical decisions. A human-centered approach is an example of a design value that would be intertwined with the 
design process. For example, in their phenomenographic study of human-centered design, Zoltowski, Oakes, and Cardella ${ }^{27}$ identified seven distinct ways that students experience (and understand) human-centered design. The most comprehensive category from this study, Empathic Design, was characterized by a very broad and integrated understanding of the stakeholders and the social, cultural, political, technical, and ethical issues associated with the design. Design knowledge was gained through a connection with end users and there was evidence of their consideration of "everyday ethics" throughout their design process.

\section{Ethics in Team Communication and Interactions}

Group communication scholars have extensively examined decision-making processes in teams, finding how team characteristics such as diversity and status differences affect team decisionmaking and performance. Individual team members can significantly affect the team's performance, the way members relate to one another, the type of information that is shared and discussed, and a number of other factors that contribute significantly to a team's functioning and decision-making ${ }^{28-32}$. This effect is even more pronounced for smaller teams of people ${ }^{33}$, such as project teams. The team's diversity can influence decision-making and performance. Researchers have found that diversity among team members has several implications. First, more diversity in terms of age and educational experience have been linked positively to team performance ${ }^{34}$. Additionally, overall, more diverse work groups often produce more flexibility, innovation, and productivity ${ }^{35-36}$. However, research also has indicated that these more diverse work groups often encounter difficulty initially in terms of group performance and functioning ${ }^{35}$.

Cultural diversity also impacts a team's interactions and decision-making processes, providing both benefits and challenges. Some obvious challenges include potential language barriers, but nonverbal cultural differences can also make team interactions more difficult. Cultural diversity can offer more opinions and perspectives on problems ${ }^{36-37}$, and could influence the team to take into consideration a wider view of the end user of the product or its functionalities. Culture can also impact the development of team norms, the quality of discussion and inclusivity of team members, and the clarity of the decision-making process.

\section{Team Network Structure and Ethical Interactions}

In understanding decision-making and ethical reasoning in teams, the role of communication becomes essential in the team context. Communication encompasses the verbal messages team members use to share information with each other, but also involves nonverbal factors (such as "body language" and seeming enthusiastic or skeptical), and the relations formed between members as they interact. Several studies have found that the way team members communicate with each other is crucial in determining how they collaborate and the success of those efforts ${ }^{38-}$ ${ }^{40}$. However, the precise role of communication in contributing to a group's success is still a matter of debate ${ }^{41}$, with calls for more extensive work analyzing these effects ${ }^{4}$.

Past research has examined ethical behavior in organizations noting its highly complex nature and many influences. For instance, scholars in organizational studies have debated about the root of unethical decision-making and behaviors, arguing whether it is a function of "bad apples" or "bad barrels" 42 , that is, whether individual characteristics or organizational and societal influences are greater contributors. More complex models have been developed to describe a 
complex interaction between these factors, such as a focus on the types of ethical issues and their "moral intensity" in determining ethical responses ${ }^{43}$. The importance of relationships among social actors in an organization or team has emerged as an essential consideration in this debate $^{44}$. However, these studies have failed to examine the decision-making process itself, focusing rather on the outcomes and net effect of these interactions.

\section{Social Network Analysis}

As indicated earlier, social network analysis (SNA) is a type of analysis that enables researchers to examine the relationships among members of a given system or group. Several elements of social network analysis are important in understanding the strength, linkages, and patterns of team networks: external structural rules (or network-level measurements) of network density; the presence of weak ties; and internal structural rules (or those that give information about the participation of each specific actor in the network) including degree centrality and individual ethical attributes. Different network structures have been found to affect employability, employee turnover, employee satisfaction, and creativity ${ }^{46-47}$. However, how such elements of team network structures affect team ethical decision making is not known. Indeed, Whitbred et al. ${ }^{9}$ recommend that "Future research should focus on establishing whether the structuration of social networks will vary depending on the nature of the organization and, if so, which structural rules would emerge as being most important in these other contexts" (p. 425) particularly for engineering design teams.

\section{Structuration Theory}

This study follows Whitbred et al.' 's approach that combines social network analysis with structuration theory. This approach enables us to examine the structure of project teams while also examining the institutional and contextual factors that contribute to team climate, and to the development of group norms that affect team interactions. Structuration accounts for the influence of institutional factors such as rules or norms of what is "acceptable" or "appropriate" behavior within a specific social context, while also affording the actors within that context agency to effect those structural influences. This theory envisions a reflexive relationship in which institutional influences constrain and enable individual activity, while individual activity reinforces these structures and shapes them over time. Network analysis provides a concrete visualization of this relationship, showing the relational patterns of individuals to both identify local structural properties and utilize these properties to help predict and explain changes in the network structure 9 .

\section{Research Design}

Our study, guided by the four research questions stated earlier, contributes to our understanding of "everyday ethics" and ethical decision-making in project teams by looking at what happens in practice during engineering design, in an undergraduate context, and with explicit attention paid to the team communicative process. The expected outcomes are as follows:

1. Findings on how "everyday" ethics is communicatively constituted in the engineering design team processes. 
2. Findings on how individual team members influence the team's organization and behaviors in team decision-making, and how those are influenced by disciplinary and cultural diversity.

3. Findings on how team ethical decision-making is shaped by the organization of the team and the interactions of the team members.

4. Findings on what characteristics of the team structure and interactions facilitate the ethical decision-making processes of the students as well as contribute to their ability to make ethical design decisions within their project teams.

The model in Figure 1 illustrates the reflexive relationship between individual and team ethical reasoning, and how network structure and interactions both complicate and shape these decisionmaking processes. Furthermore, it demonstrates the influence of the design and institutional contexts, as well as individual and team factors and characteristics.

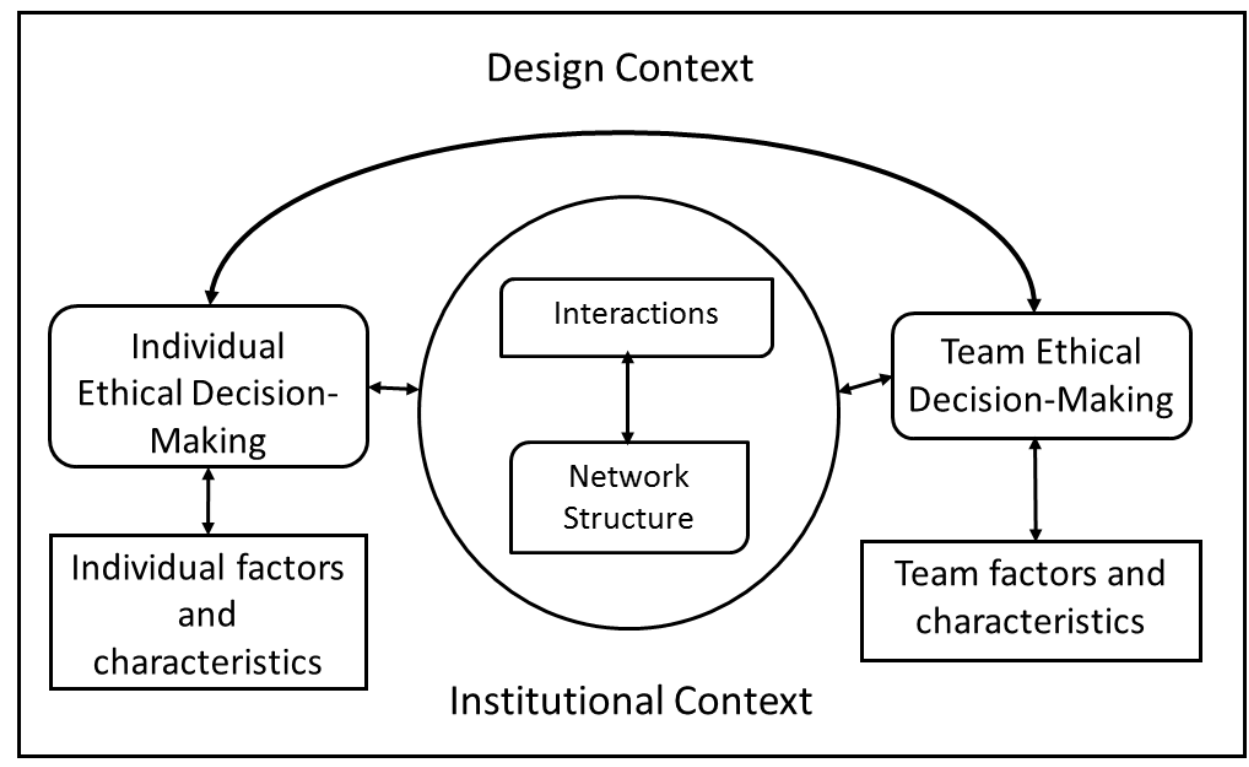

Figure 1. Relationship between different components

\section{Methods}

This study employs a mixed methodological approach ${ }^{48-49}$ in order to gain a comprehensive understanding of the issues raised above, with emphasis on a qualitative approach to the study of project teams. Combining multiple and complementary data sources and paradigms provide "strengths that offset the weaknesses of both quantitative and qualitative research" ${ }^{48}$ " (p. 12). This study utilizes a convergent (quan/QUAL) parallel design ${ }^{48}$. The qualitative data sources that include in-depth semi-structured interviews and videotaped participatory observations will let us explore how team members perceive, experience and understand ethics (the "everyday ethics") of design; while the Social Network Analysis (SNA) component of the study will let us explore team structural characteristics and their impact on those perceptions and the team's overall discussions and decision-making. 


\section{Participants and Recruitment Strategies}

We have purposefully selected four diverse project teams within the EPICS program, a servicelearning design program at Purdue University. In this program, teams of undergraduates partner with local or global not-for-profit community organizations to define, design, build, test, deploy, and support engineering-centered projects that significantly improve the organization's ability to serve the community. There are larger teams that represent the class division that generally have the same project partner, as well as project teams that share a common design goal. Students can participate multiple semesters; teams typically have a mix of returning and new students. Students take on different roles, such as project manager, design lead, financial officer, and project partner liaison. To maximize the use of social network analysis, we selected four project teams within four separate classes comprised of 2-6 project teams each to follow each semester that consist of 70-80 individuals total, including instructors (advisors) and graduate teaching assistants. This format enables us to examine relations within the specific project teams, as well as how project teams interact with others in the same class. Project teams ranged from 3-9 members. We purposefully sampled project teams that are culturally diverse as well as those which are multidisciplinary. We are conducting data collection at the end of two consecutive semesters. During the second semester, we are purposefully sampling a limited number of teams with various levels of membership change from the first semester so that we can examine how the team structure and interactions change as team membership changes.

\section{Observations}

To enable analysis of the interactions of the team members during their decision-making discussions, we observed several meetings (designated class period, outside project meetings) of the selected project teams. We are following the guidelines for conducting naturalistic study ${ }^{50}$, observing the practical accomplishments of everyday meetings to understand how the team communicatively constitutes ethics in the decision-making process. To allow for rich analysis of these interactions as well as repeated viewing of the interactions as they happen, we will also use video recording to capture these interactions.

Research memos of the observations are completed and will be used to provide insight into how team members negotiate value differences or conflicts, what role members seemed to occupy during these discussions, what issues were raised and how the team treated these issues, and other relevant interactions that emerge from this examination. We will examine the flow of interaction among participants and how what they say and do maintains or alters the ethical decision making.

\section{Social Network Analysis}

The social network analysis ${ }^{51}$ (SNA) was administered to all members of the four classes, allowing us to assess a large network (the class) and how the project teams within it interact. The survey contained two sections: (a) a sociometric instrument that provides a complete list of all the members of a project team and asks the participant to relate their communicative relationships with them ${ }^{51}$, and (b) relevant demographic information including age, gender, ethnic/race category, perceived role in the group, and other important factors that may be considered at various points in the analysis. Although data collection occurs once per semester, 
the interview and survey asks participants to discuss how the interactions have changed over the course of the semester.

Using UCINET, a social network analysis tool, we are examining two levels of social network measurements, those that describe the network as a whole (network density and the presence of weak ties) and those that give information about the participation of each specific actor in the network (degree centrality and individual ethical attributes). These analyses illustrate whether certain individuals in the project teams have more influence in specific relational contexts. These results will be examined to identify correlations between certain network or actor measurements and the emergent network structure these qualities produce. These results will be examined with and in comparison to the results of the qualitative data analysis and in light of the theoretical framework of this study to identify implications of their relationships.

\section{Moral Reasoning as Individual Attributes}

In addition to traditional network measurements, we are utilizing the scores from our measure of individual ethical reasoning instrument ${ }^{52}$ of individuals to visualize the "ethical network" structure that emerges within the project teams during the second data collection. By treating an individual's score as an attribute, we will be able to run analyses to examine the relationships and structures that emerge among team members according to this attribute--for example, do high moral reasoning individuals operating at a post-conventional level cluster together? Do they frequently emerge as central in the network, or have positions of particular prominence or influence? We will be able to examine whether an individual's influence extends to ethical decision making such that team climates, and the discussions surrounding decision-making on the team, reflect their positions.

\section{Interviews}

To date we have conducted 66 in-depth interviews that probe deeply into the team and design process. There are two sections to the interviews. The first is a semi-structured interview with questions about team member interactions, design decisions, and considerations the participant had as well as any considerations that were raised by other team members. These questions were adapted from the interview protocol used in our previous project and follow these themes:

- asking participants to recall and describe two or three decisions their team has made thus far in the project

- asking them to describe as they see it the design process their team has followed, including asking them to chart out the choices the team has made along a timeline

- asking about team member interactions, such as who the participant would go to for advice, who speaks up most often in team discussions, what is the tone and atmosphere of decision-making discussions, and how the participant perceives the roles and qualifications of each member of their project team

The second part of the interview explores the participants' responses to the SNA survey, probing why they indicated the people they did and differences in responses to the categories. Interviews are being transcribed and de-identified to protect participants' identities. We have begun analysis of the interview data using a typological analysis approach ${ }^{53-54}$. Initial codes were generated from the individual ethical reasoning and team culture instruments we are developing. 
As we analyze this interview data, any themes or ideas that seem prevalent across respondents are being added. We are comparing and contrasting findings emerging from data with interdisciplinary research to uncover regular patterns of communication that indicate how ethics is communicatively constituted by team members, team norms for ethical decision making procedures, and other aspects that are part of team processes.

\section{Future Work}

We are conducting our second round of data collection this semester (Spring 2015), following the same four project teams that were observed, interviewed, and completed the social analysis network (SNA) survey during the Fall, 2014 semester. A third data collection is planned for Fall, 2015. Data analyses and integration of the interviews, SNA surveys, and observation are ongoing.

\section{Summary}

This study builds on prior NSF-funded work and a broad literature from engineering education and the social sciences to address the compelling issue of ethical awareness and ethical reasoning within diverse design teams. Design is a central function of engineering and ethics is often learned within undergraduate design courses where many ethical decisions are made through smaller more frequent design decisions and involve interactions with team members. This project fills a gap in ethics scholarship by examining how everyday ethical decision-making is integrated systematically in the design processes and interactions of diverse design teams. It examines ongoing ethical decision-making interactions and structures that occur during the everyday work of diverse design teams and lays the foundation to create models that can inform curriculum development.

The findings of this research have potential impacts across engineering education. Today's technology provides the engineering community with an enormous opportunity to positively impact society if applied appropriately. Today's global society adds complexity to the social and ethical issues that need to be addressed by designers and professionals as technology is applied to address needs. Better understanding the development of ethical reasoning within diverse design teams as they make design decisions can greatly enhance the way engineers and other technical professionals learn key attributes called for by ABET, the NAE's Engineer of 2020, and industry stakeholders. Designing curricular experiences to equip engineering students to address ethical and societal challenges requires developmental ethical models that account for team interactions as they produce or constitute the nature, meaning, and outcomes of ethical decision making, structures that emerge as design teams operate and that shape and are shaped by ethical decision-making behaviors, and how these interactions and structures are influenced by different disciplines, cultures, and organizational climates. The project also introduces crossdisciplinary research methods from communication into engineering education.

\section{Acknowledgements}

This work was made possible by grants from the National Science Foundation (EEC-1429114) and Purdue Research Foundation. Any opinions, findings, and conclusions or recommendations 
expressed in this material are those of the authors and do not necessarily reflect the views of the National Science Foundation or Purdue University.

\section{References}

[1] Larson, J. R. (2007). Deep diversity and strong energy modeling the impact of variability in members' problem-solving strategies on group problem-solving performance. Small Group Research, 38: 413-436.

[2] Postmes, T., Spears, R., \& Cihangir, S. (2001). Quality of decision making and group norms. Journal of personality and social psychology, 80(6), 918-930. doi: 10.1037//0022-3514.80.6.918

[3] Reimer, T., Reimer, A., \& Czienskowski, U. (2010). Decision-making groups attenuate the discussion bias in favor of shared information: A meta-analysis. Communication Monographs, 77(1), 121-142. doi: 10.1080/03637750903514318

[4] Seibold, D., Hollingshead, A., \& Yoon, K. (2014). Embedded teams and embedding organizations. In L. Putnam \& D. Mumby (Eds.), The SAGE handbook of organizational communication: Advances in theory, research, and methods (pp. 327-349). Los Angeles, CA: Sage.

[5] Zoltowski, C., Buzzanell, P., Oakes, W., \& Kenny, M. (2013). A Qualitative Study Exploring Students' Engineering Ethical Reflections and their Use in Instrument Validation, Proceedings of the 2013 Frontiers in Education Conference, Oklahoma City, OK, October 2013.

[6] Fyke, J., \& Buzzanell, P.M. (2013). The ethics of conscious capitalism: Wicked problems in leading change and changing leaders. Human Relations, 66, 1619-1643. doi: 10.1177/0018726713485306Trevino, L. K. (1986). Ethical decision making in organizations: A person-situation interactionist model. The Academy of Management Review, 11(3), 601-617.

[7] Trevino, L. K., Butterfield, K. B., \& McCabe, D. L. (1998). The ethical context in organizations: Influences on employee attitudes and behaviors. Business Ethics Quarterly, 8(3), 447-476.

[8] Whitbred, R., Fonti, Fabio, Steglich, C., \& Contractor, N. (2011) From Microactions to Macrostructure and Back: A structurational approach to the evolution of organizational networks. Human Communication Research 37: 404-433.

[9] Rest, J., Narvaez, D., Bebeau, M., \& Thoma, S. (1999). A neo-Kohlbergian approach: The DIT and schema theory. Educational Psychology Review, 11 (4), 291-324.

[10] Rest, J., Thoma, S., Narvaez, D., \& Bebeau, M. (1997). Alchemy and beyond: Indexing the defining issues test. Journal of Educational Psychology, 89 (3), 498-507.

[11] Van de Poel, I., \& Royakkers, L. (2011). Ethics, technology, and engineering: An introduction. Oxford, UK: Wiley-Blackwell.

[12] Van de Poel, I. \& Verbeek, P. (2006). Editorial: Ethics and engineering design. Science, Technology \& Human Values, 31(3), 223-236.

[13] Lloyd, P., \& Busby, J. (2003). “Things that went well - no serious injuries or deaths": ethical reasoning in a normal engineering design process. Science and Engineering Ethics, 9(4), 503-516.

[14] Verbeek, P. (2006). Materializing morality: Design ethics and technological mediation. Science, Technology \& Human Values, 31(3), 361-380.

[15] Atman, C. J., Adams, R. S., Cardella, M. E., Turns, J., Mosborg, S., \& Saleem, J. J. (2007). Engineering design processes: A comparison of students and expert practitioners. Journal of Engineering Education, 96(4), 359 379.

[16] Bennett, P. (2006). Listening lessons: Make consumers part of the design process by tuning in. Point, March 2006. Accessed online May 10, 2009.

[17] EPICS Design Process: http://epics.ecn.purdue.edu/resources/EPICS\%20Design\%20Process.pdf. Accessed May 10, 2009.

[18] Ullman, D. G. (2003). The Mechanical Design Process, Third Edition. New York: McGraw-Hill Higher Education.

[19] Mosborg, S., Adams, R. Kim,, R. Atman, C.J., Turns, J. and Cardella, M. (2005). Conceptions of the Engineering Design Process: An Expert Study of Advanced Practicing Professionals, Proceedings of the Annual American Society of Engineering Education Conference, Portland, Oregon. 
[20] Crismond, D. P., \& Adams, R. S. (2012). The Informed Design Teaching and Learning Matrix. Journal of Engineering Education, 738-797.

[21] Bucciarelli, L. L. (1996). Designing engineers. Cambridge: MIT Press.

[22] Dym, C. L., Agogino, A. M., Eris, O., Frey, D. D., \& Leifer, L. J. (2005). Engineering design thinking, teaching, and learning. Journal of Engineering Education, 94, 103-120.

[23] Krippendorff, K. (2006). The semantic turn: A new foundation for design. Boca Raton, FL: CRC Press Taylor \& Francis Group.

[24] Hoffman, R. R., Feltovich, P. J., Ford, K. M., Woods, D. D., Klein, G., \& Feltovich, A. (2002). A rose by any other name...would probably be given an acronym. IEEE Intelligent Systems, 17(4), 72-80.

[25] Zhang, T., \& Dong, H. (2008). Human-centred design: an emergent conceptual model. Include2009. Available online http://www.hhc.rca.ac.uk/2084/all/1/ proceedings.aspx. Accessed 3/28/2010.

[26] Zoltowski, C. B., Oakes, W. C., Cardella, M. (2012). Students' Ways of Experiencing Human-Centered Design, Journal of Engineering Education, 101(1).

[27] Bain, P. G., Mann, L. \& Pirola-Merlo, A. (2001) "The Innovation Imperative The Relationships Between Team Climate, Innovation, and Performance in Research and Development Teams." Small Group Research 32.1 (2001): 55-73.

[28] Barnlund, D. C., \& Haiman, F. S. (1960). The dynamics of discussion (pp. 275-292). Boston: Houghton Mifflin.

[29] Dahlin, K. B., Weingart, L. R., \& Hinds, P. J. (2005). Team diversity and information use. Academy of Management Journal, 48(6), 1107-1123.

[30] Gibb, J. R. (1978). Defensive communication. Shared Experiences in Human Communication, 44.

[31] Karakowsky, L., \& McBey, K. (2001). Do my contributions matter? The influence of imputed expertise on member involvement and self-evaluations in the work group. Group \& Organization Management, 26(1), 7092.

[32] Henley, A. B., \& Price, K. H. (2002). Want a better team? Foster a climate of fairness. The Academy of Management Executive, 16(3), 153-154.

[33] Kearney, E., Gebert, D., \& Voelpel, S. C. (2009). When and how diversity benefits teams: the importance of team members' need for cognition. Academy of Management Journal, 52(3), 581-598.

[34] Fritz, K. (1999) The Diversity Dilemma: Dealing with Difference. Presented at the Vocation of Lutheran Institution Conference, Selinsgrove, PA.

[35] Miura, A., \& Hida, M. (2004). Synergy between diversity and similarity in group-idea generation. Small Group Research, 35(5), 540-564.

[36] McLeod, P. L., Lobel, S. A., \& Cox, T. H. (1996). Ethnic diversity and creativity in small groups. Small group research, 27(2), 248-264.

[37] de Moura, G. R., Leader, T., Pelletier, J., \& Abrams, D. (2008). Prospects for group processes and intergroup relations research: A review of 70 years' progress. Group Processes \& Intergroup Relations, 11(4), 575-596.

[38] Hirokawa, R. Y., Degooyer, D., \& Valde, K. (2000). Using narratives to study task group effectiveness. Small Group Research, 31(5), 573-591.

[39] Salazar, A. J. (1996). Ambiguity and Communication Effects on Small Group Decision-Making Performance. Human Communication Research, 23(2), 155-192.

[40] Meyers, R. A., \& Seibold, D. R. (2009). Making foundational assumptions transparent: Framing the discussion about group communication and influence. Human Communication Research, 35(2), 286-295.

[41] Trevino, L. K. \& Youngblood, S. A. (1990) Bad apples in bad barrels: A causal analysis of ethical decisionmaking behavior. Journal of Applied Psychology, 75: 378-385.

[42] Jones, T. M. (1991) Ethical decision making by individuals in organizations: An issue-contingent model. Academy of Management Review, 16: 366-395.

[43] Brass, D. J., Butterfield, K. D., \& Skaggs, B. C. (1998). Relationships and unethical behavior: A social network perspective. Academy of Management Review, 23(1), 14-31.

[44] Monge, P. R., \& Eisenberg, E. M. (1987). 10 Emergent Communication Networks. Handbook of Organizational Communication: an interdisciplinary perspective, 304.

[45] Granovetter, M. (1983). The strength of weak ties: A network theory revisited. Sociological Theory, 1(1), 201233.

[46] Krackhardt, D., \& Hanson, J. R. (1993). Informal networks. Harvard Business Review, 71(1993), 104-11.

[47] Jung, T., Scott, T., Davies, H. T. O., Bower, P., Whalley, D., McNally, R. \& Mannion, R. (2009) Instruments for Exploring Organizational Culture: A Review of the Literature. New Ideas for Improving Public Administration. 
[48] Creswell, J. W., and V. L. Plano Clark. (2011). Designing and Conducting Mixed Methods Research (Second Edition). Thousand Oaks, CA: SAGE Publications.

[49] Yauch, C., \& Steudel, H. (2003). Complementary use of qualitative and quantitative cultural assessment methods. Organizational Research Methods, 6, 465-481.

[50] Lindlof, T. R. \& Taylor, BC (2002). Qualitative communication research methods.

[51] Wasserman, S., \& Faust, K. (1994) Social Network Analysis: Methods and applications. Cambridge, England: Cambridge University Press.

[52] Zhu, Q., Zoltowski, C. B., Feister, M. K., Buzzanell, P. M., Oakes, W. C. \& Mead, A. D. (2014). The development of an instrument for assessing individual ethical decisionmaking in project-based design teams: Integrating quantitative and qualitative methods. To appear in Proceedings of the American Society for Engineering Education Annual Conference \& Exposition, Indianapolis, IN.

[53] Hatch, J. A. (2002). Doing qualitative research in education settings. Albany, NY: State University of New York Press.

[54] Ayres, L., \& Knafl, K. (2008). Typological analysis. In L. Given (Ed.), The SAGE encyclopedia of qualitative research methods. (pp. 901-902). Thousand Oaks, CA: SAGE Publications, Inc. doi: 10.4135/9781412963909.n472 